\title{
Design of Symmetrical Class E Power Amplifiers for Very Low Harmonic-Content Applications
}

\author{
Siu-Chung Wong, Member, IEEE, and Chi K. Tse, Senior Member, IEEE
}

\begin{abstract}
Class E power amplifier circuits are very suitable for high efficiency power amplification applications in the radio-frequency and microwave ranges. However, due to the inherent asymmetrical driving arrangement, they suffer significant harmonic contents in the output voltage and current, and usually require substantial design efforts in achieving the desired load matching networks for applications requiring very low harmonic contents. In this paper, the design of a Class $E$ power amplifier with resonant tank being symmetrically driven by two Class $E$ circuits is studied. The symmetrical Class $\mathbf{E}$ circuit, under nominal operating conditions, has extremely low harmonic distortions, and the design of the impedance matching network for harmonic filtering becomes less critical. Practical steady-state design equations for Class $\mathbf{E}$ operation are derived and graphically presented. Experimental circuits are constructed for distortion evaluation. It has been found that this circuit offers total harmonic distortions which are about an order of magnitude lower than those of the conventional Class E power amplifier.
\end{abstract}

Index Terms-Class $\mathrm{E}$ power amplifier, push-pull amplifier, resonant converter, harmonic distortion.

\section{INTRODUCTION}

$\mathbf{C}$ LASS E zero-voltage-switching (ZVS) resonant power amplifiers are widely regarded as a very efficient type of converters [1]-[6], which have found applications in high-frequency power amplification, and in particular radio-frequency (RF) transceiver systems [7]-[10]. In the original single-ended design, the Class E power amplifier is driven asymmetrically. The energy of higher harmonics generated in the amplifier results in harmonic distortions in the output sine voltage and current, which must be filtered for some applications. For instance, radio transmitters' output harmonic contents must meet national or international regulatory requirements, e.g., all harmonics being $65 \mathrm{~dB}$ or more below the carrier. Any practical filtering method, however, incurs a penalty on the overall efficiency and the design cost. Low-distortion CMOS complementary Class E power amplifiers have been proposed [11], [12], in which two identical resonant circuits are used. This design, however, poses a practical problem of matching inductors and capacitors.

Recently, a symmetrically driven push-pull Class E amplifier has been proposed for high power applications, with its output

Manuscript received August 16, 2004; revised January 27, 2005. This work was supported by the Hong Kong Research Grants Council under Competitive Earmarked Research Grant PolyU 5219/04E. This paper was recommended by Associate Editor A. Ioinovici.

The authors with the Department of Electronic and Information Engineering, Hong Kong Polytechnic University, Hong Kong (e-mail: enscwong@polyu.edu.hk; encktse@polyu.edu.hk).

Digital Object Identifier 10.1109/TCSI.2005.851723 voltage doubled over the original Class E circuit [13]. In addition to the high power capability and high efficiency, this symmetrically driven amplifier offers very low harmonic distortions ${ }^{1}$ and hence can ease the design of the matching filter for applications requiring sinewave outputs with low harmonic contents. For the original Class $\mathrm{E}$ amplifier, calculations of harmonic amplitudes are given in Sokal and Raab [14]. However, very little work has been done to analyze the operation and to evaluate the harmonic contents of the outputs of the symmetrically driven Class E amplifier, despite the many potential advantages it offers. Theoretically, the push-pull configuration provides perfect cancellation of all even harmonics, if the "push" and "pull" stages are identical and their drive waveforms are exactly $180^{\circ}$ apart. Thus, the symmetrically driven push-pull Class E amplifier is expected to have a negligibly small amount of even harmonics. However, the odd harmonics may still be significant. In this paper, we will derive the nominal operating conditions and design equations of this circuit, and evaluate its distortion performance experimentally.

\section{PRINCIPLE OF OPERATION}

The symmetrically driven Class E circuit under study is shown in Fig. 1. It consists of an $L C R$ parallel resonant tank which is driven symmetrically by two MOSFET switches $Q_{1}$ and $Q_{2}$. As in typical Class E operation, the body diodes of the switches, $D_{1}$ and $D_{2}$, form a useful part of the circuit. Here, we note that the LCR resonant tank effectively serves as a harmonic filter whose characteristic can be readily derived. The resistor $R$ is the ac output load of the power amplifier. In most RF applications, resistor $R$ has to be "transformed" to the actual RF load (normally $50 \Omega$ ) via an impedance matching network. In addition, this impedance matching network can also be designed to provide the necessary harmonic filtering. Literature abounds with filter design procedures for the purposes of harmonic filtering and load matching [15]-[18]. Furthermore, if the load is grounded at one side and to be fed from an unbalanced line, a balun can be used to provide the interface with the amplifier [19]; and if the output of the amplifier is balanced to ground and feeding a balanced load through a balanced transmission line, inductor $L$ should be split in two halves, each to be placed at each side of capacitor $C$.

The basic operating principles of the Class $\mathrm{E}$ amplifier have been described previously in Sokal and Sokal [1] and Sokal [2]. For the symmetrically driven Class E amplifier shown in Fig. 1, the switches are driven on and off alternately within each of

\footnotetext{
${ }^{1}$ The harmonic distortions being referred to in this paper are generated by the amplifier, and should not be confused with the distortions arising from some nonlinear input-to-output transfer characteristics of the amplifier.
} 


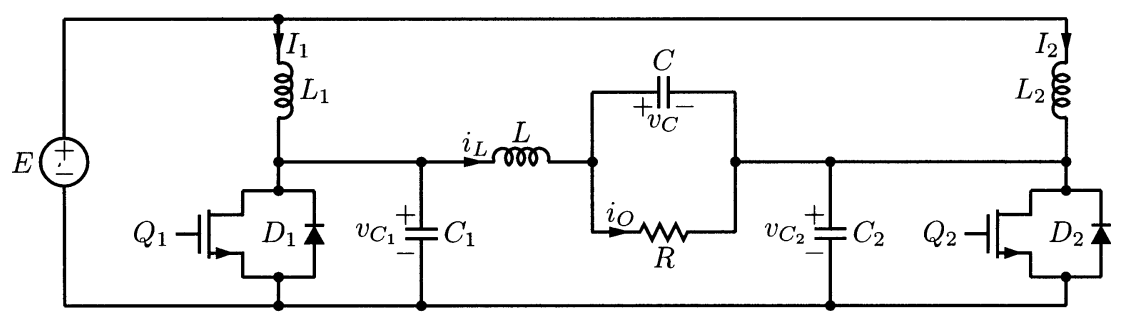

Fig. 1. Symmetrically driven Class E power amplifier.

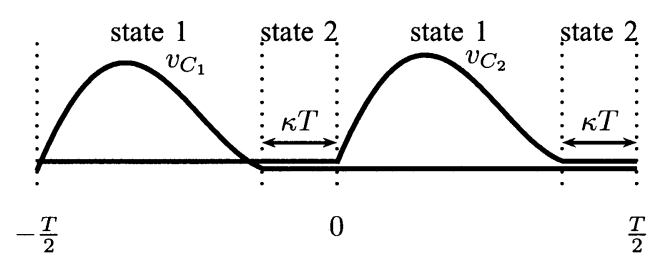

(a)

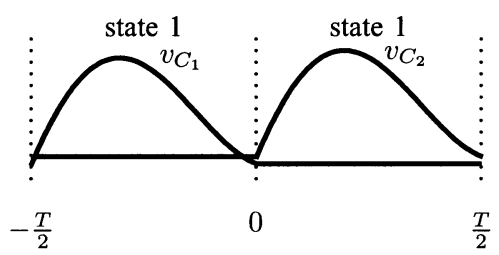

(b)

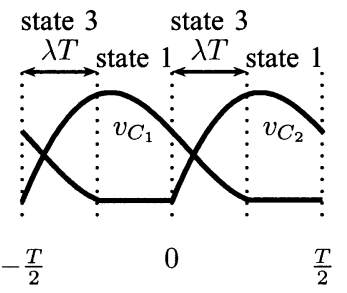

(c)

Fig. 2. Three possible operating modes of the symmetrically driven Class $\mathrm{E}$ power amplifier. (a) Operating mode 1. (b) Operating mode 2. (c) Operating mode 3.

the half operating period $T / 2(T=1 / f=2 \pi / \omega)$. The duty cycle of the switches are adjusted so as to achieve ZVS. The components are chosen to satisfy the symmetry conditions, i.e., $Q_{1}=Q_{2}\left(D_{1}=D_{2}\right), L_{1}=L_{2}$, and $C_{1}=C_{2}$. Thus, the waveforms of $I_{1}$ and $v_{C_{1}}$ should resemble those of $I_{2}$ and $v_{C_{2}}$, respectively, but with a phase shift of $\omega T / 2$, i.e., $\pi$. The parallel resonant tank is then driven by a nearly sinusoidal voltage given by $v_{T}=v_{C_{1}}-v_{C_{2}}$.

Depending upon the driving frequency $\omega$, three sets of steady-state waveforms are shown in Fig. 2, denoted as operating modes 1, 2, and 3. For modes 1 and 3, a total of four switching states can be found, and for mode 2 , two switching states can be found. If we take polarity inversion for $v_{C}$ and $i_{L}$ and interchange variables $L_{1}, v_{C_{1}}, I_{1}$ with $L_{2}, v_{C_{2}}, I_{2}$, respectively, the waveforms for the second half period simply resemble those of the first half period. Thus, we need to consider only the half switching period. The circuit for states 1,2 , and 3 are given in Figs. 3-5, respectively. It should be noted that Fig. 2(b) is a special case of Fig. 2(a) and (c) with $\kappa=0$ and $\lambda=0$, respectively. It should be obvious that operations in

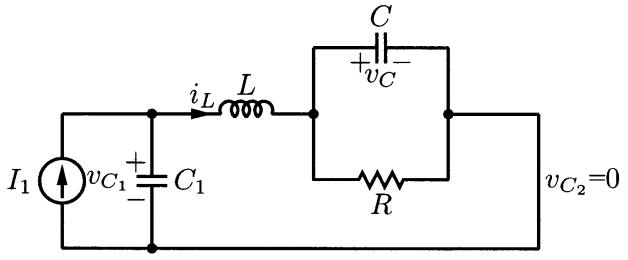

(a)

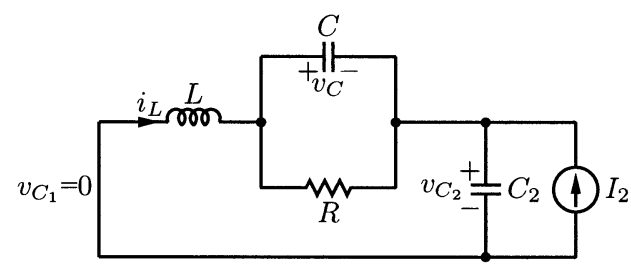

(b)

Fig. 3. State 1 of the symmetrically driven Class E amplifier.

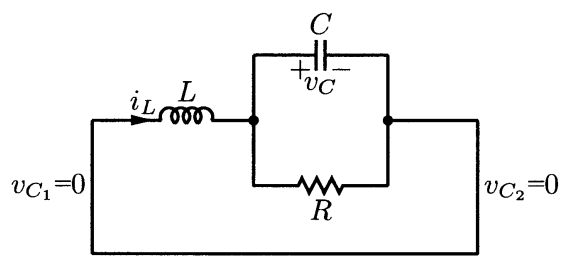

Fig. 4. State 2 of the symmetrically driven Class E amplifier.

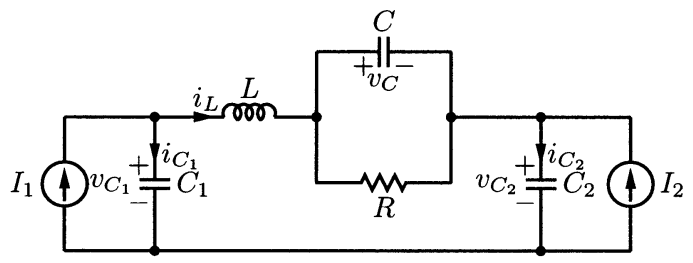

Fig. 5. State 3 of the symmetrically driven Class E amplifier.

the vicinity of operating mode 2, as shown in Fig. 2(b), should give the lowest harmonic distortion. In the subsequent analysis, we will focus on this case.

Analysis procedures for such Class E ZVS power amplifiers using a fundamental frequency component method can be found in Kazimierczuk and Czarkowski [20]. The method, however, is not applicable in finding the conditions for Class E operation where the driving voltage $v_{T}(t)$, i.e., $v_{C_{1}}-v_{C_{2}}$, is having a high harmonic content. The fundamental frequency component method is therefore applicable only to the resonant tank circuit. The basic assumptions are as follows. 
1) The transistors, capacitors and inductors are ideal and lossless.

2) $I_{1}$ and $I_{2}$ are constant current sources. They are equal and interchangeable in the steady state.

3) The fundamental component of the tank inductor current is assumed to be

$$
\begin{aligned}
i_{L}(t) & =I_{L} \sin (\omega t+\phi)+\text { higher harmonics } \\
& \approx I_{L} \sin (\omega t+\phi)
\end{aligned}
$$

where $I_{L}$ and $\phi$ are amplitude and phase, respectively, of $i_{L}$.

Referring to the circuits shown in Figs. 3-5 for the various switching states, the steady-state equations for operating modes 1,2 and 3 are shown in (2) at the bottom of the page, where $\kappa<(1 / 2), \lambda<(1 / 2)$, the notation $x \in[a, b)$ is equivalent to $a \leq x<b$, and $\cup$ denotes union of two sets.

The capacitor voltage $v_{C_{1}}$ can be found by integrating (2), i.e., as shown in (3) at the bottom of the page. The boundary condition for ZVS can be found by solving

$$
\begin{array}{cccc} 
& \text { Mode 1 } & \text { Mode 2 } & \text { Mode 3 } \\
v_{C_{1}}(\sigma T)=0, \quad \text { for } \sigma=-\kappa & 0 & \lambda
\end{array}
$$

which gives

$$
I_{L} \cos (\pi \sigma)(-\cos (\pi \sigma+\phi))=I_{1} \pi\left(\frac{1}{2}+\sigma\right)
$$

where $\cos (\pi \sigma)>0$ for $|\sigma|<(1 / 2)$ and $-\cos (\pi \sigma+\phi)>0$ for some $\pi \sigma+\phi$.
Equation (5) can be referred to as "suboptimum" condition in Class $\mathrm{E}$ amplifiers, where $i_{C_{1}}(\sigma T)<0$ is utilized to achieve soft switching. The anti-parallel diode $D_{1}$ of transistor $Q_{1}$ conducts while $v_{C_{1}}$ has reversed its polarity. The voltage across $Q_{1}$ will then be held at a low level equal to the diode drop. Transistor $Q_{1}$ is then turned on by the control circuit to take over the current of the body diode and keep the capacitor $v_{C_{1}}$ at near $0 \mathrm{~V}$. The switching loss can be further reduced if the current at the switching instant is also low. If the condition of zero-current-switching (ZCS), $i_{C_{1}}(\sigma T)=0$, is also enforced, we have the nominal operation. This corresponds to

$$
I_{L} \sin (2 \pi \sigma+\phi)=I_{1}
$$

Combining (5) and (6), we obtain a condition for a nominal operation, i.e.,

$$
-\pi\left(\frac{1}{2}+\sigma\right)[\tan (\pi \sigma+\phi)+\tan (\pi \sigma)]=1 .
$$

The resonant tank is driven by $v_{T}(t)=v_{C_{1}}(t)-v_{C_{2}}(t)$ as shown in Fig. 2, which is given by (8) shown at the bottom of the page. Putting (5) in (3) gives (9) shown at the bottom of the page, where $A=\left(I_{1} \pi / \omega C_{1}\right)$ and $B=((1 / 2)+\sigma) /(\cos (\pi \sigma+\phi) \cos (\pi \sigma))$. Using Fourier analysis, it can be shown that the harmonic components of (8) is lowest when operating near Mode 2, i.e., $\sigma \approx 0$. In the subsequent analysis, we will assume $\sigma=0$.

Using the fundamental frequency component method [20], an equivalent circuit for the converter is shown in Fig. 6. This equivalent circuit is driven by a sinusoidal source $i(\omega)=(4 / \pi) I_{1} \sin (\omega t)$. At nominal operation, the magnitude

$$
\begin{aligned}
& i_{C_{1}}(t)=\left\{\begin{array}{lllr}
I_{1}-i_{L}(t), & \text { Mode } 1 & \text { Mode } 2 & \text { Mode } 3 \\
0, & t \in\left[-\frac{T}{2},-\kappa T\right) & {\left[-\frac{T}{2}, 0\right)} & {\left[-\frac{T}{2}, \lambda T\right)} \\
t \in\left[-\kappa T, \frac{T}{2}\right) & {\left[0, \frac{T}{2}\right)} & {\left[\lambda T, \frac{T}{2}\right)}
\end{array}\right.
\end{aligned}
$$

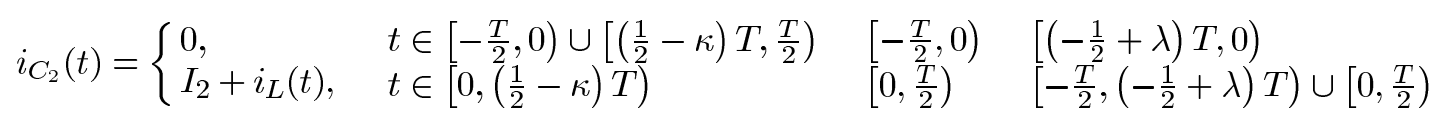

$$
v_{T}(t)= \begin{cases}v_{C_{1}}(t), & \text { state } 1 \text { and } t \in\left[-\frac{1}{2} T, 0\right) \\ 0, & \text { state } 2 \text { and } t \in[-\kappa T, 0) \\ v_{C_{1}}(t)-v_{C_{1}}\left(t+\frac{1}{2} T\right), & \text { state } 3 \text { and } t \in\left[-\frac{1}{2} T,\left(-\frac{1}{2}+\lambda\right) T\right) \\ -v_{T}\left(t-\frac{1}{2} T\right), & t \in\left[0, \frac{1}{2} T\right)\end{cases}
$$

Mode $1 \quad$ Mode 2 Mode 3

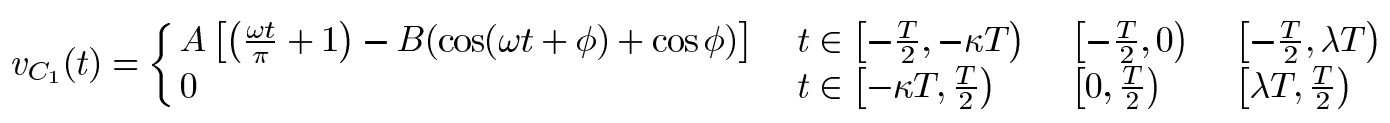




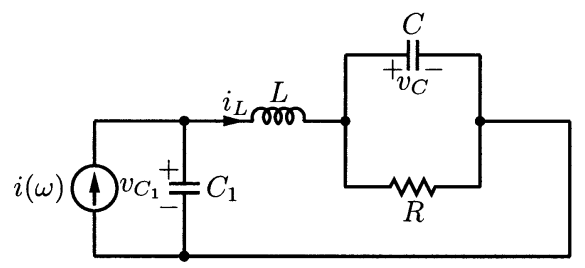

Fig. 6. Equivalent circuit based on the fundamental frequency component method.

and phase of the inductor current of the parallel resonant tank at $\omega$ are given as

$$
\begin{aligned}
& \left(\frac{I_{L}}{I_{1}}\right)^{2}(\omega) \\
& \quad=\left(\frac{4}{\pi}\right)^{2} \frac{\left[\left(Q_{L} \omega\right)^{2}+\omega_{o}^{2}\right]\left(\omega_{o}^{2}-\omega_{1}^{2}\right)^{2}}{\left[\omega Q_{L}\left(\omega^{2}-\omega_{1}^{2}\right)\right]^{2}+\left[\omega_{o}\left(\omega^{2}-\omega_{1}^{2}+\omega_{o}^{2}\right)\right]^{2}}
\end{aligned}
$$

and

$$
\begin{aligned}
& \tan (\phi(\omega)) \\
& \quad=\frac{Q_{L} \omega \omega_{o}^{3}}{Q_{L}^{2} \omega^{2}\left(\omega^{2}-\omega_{1}^{2}\right)+\omega_{o}^{2}\left(\omega^{2}-\omega_{1}^{2}+\omega_{o}^{2}\right)}
\end{aligned}
$$

respectively, where

$$
\begin{aligned}
Z_{o} & =\sqrt{\frac{L}{C}} \\
Q_{L} & =\omega_{o} C R \\
\omega_{o} & =\frac{1}{\sqrt{L C}} \\
\omega_{1} & =\frac{1}{\sqrt{L \frac{C C_{1}}{C+C_{1}}}} .
\end{aligned}
$$

Conversely, the component values of the resonant tank are represented as

$$
\begin{aligned}
L & =\frac{Z_{o}}{\omega_{o}} \\
C & =\frac{1}{\omega_{o} Z_{o}} \\
C_{1} & =\frac{1}{\omega_{o} Z_{o}\left[\left(\frac{\omega_{1}}{\omega_{o}}\right)^{2}-1\right]} \\
R & =Q_{L} Z_{o} .
\end{aligned}
$$

Combining (6), (7), (8), and (9), the nominal Class E operation point for mode 2 can be found by solving the following equations numerically.

$$
\begin{aligned}
-\frac{2}{\pi} & =\tan (\phi(\omega)) \\
& =\frac{Q_{L} \omega \omega_{o}^{3}}{Q_{L}^{2} \omega^{2}\left(\omega^{2}-\omega_{1}^{2}\right)+\omega_{o}^{2}\left(\omega^{2}-\omega_{1}^{2}+\omega_{o}^{2}\right)} \\
\frac{2^{2}}{2^{2}+\pi^{2}} & =\frac{1}{\sin ^{2}(\phi(\omega))} \\
& =\left(\frac{4}{\pi}\right)^{2} \frac{\left[\left(Q_{L} \omega\right)^{2}+\omega_{o}^{2}\right]\left(\omega_{o}^{2}-\omega_{1}^{2}\right)^{2}}{\left[\omega Q_{L}\left(\omega^{2}-\omega_{1}^{2}\right)\right]^{2}+\left[\omega_{o}\left(\omega^{2}-\omega_{1}^{2}+\omega_{o}^{2}\right)\right]^{2}} .
\end{aligned}
$$

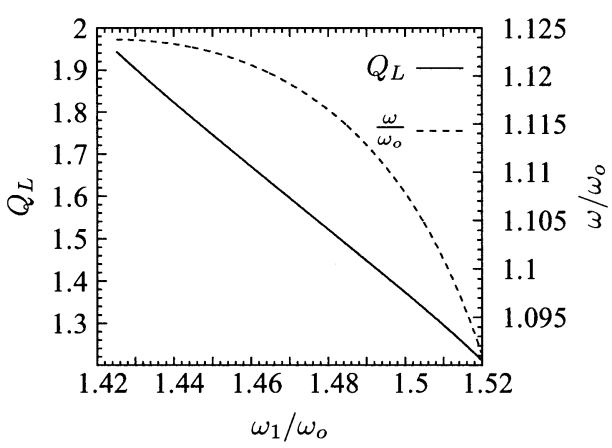

Fig. 7. Quality factor and switching frequency design curves for nominal operation of the symmetrically driven Class E amplifier.

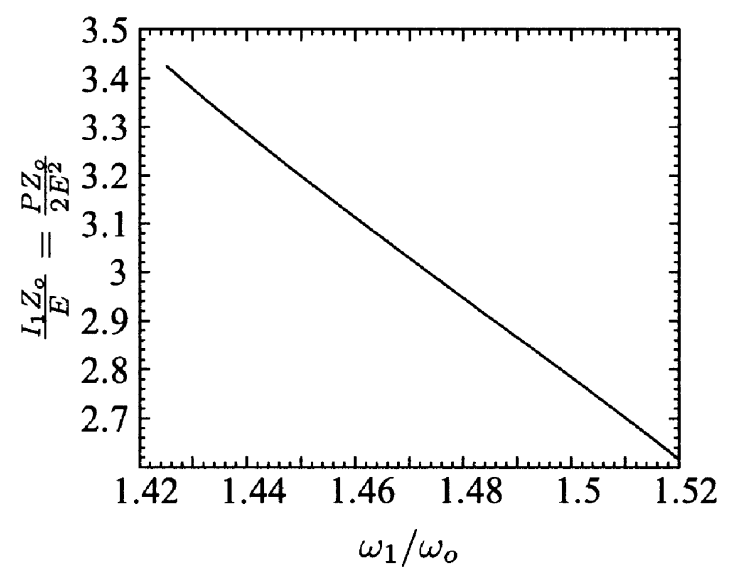

Fig. 8. Normalized input current design curve for nominal operation of the symmetrically driven Class E amplifier.

In the steady state, the volt-time product of $L_{1}$ should be zero, i.e.,

$$
2 E=\frac{2}{T} \int_{-\frac{T}{2}}^{0} v_{C_{1}}(t) d t .
$$

Solving (22), we obtain

$$
\frac{\frac{\pi \omega}{\omega_{o}}}{\left(\frac{\omega_{1}}{\omega_{o}}\right)^{2}-1}=\frac{I_{1} Z_{o}}{E}=\frac{P Z_{o}}{2 E^{2}}
$$

where $P$ is the power given by $P=2 I_{1} E$. Fig. 7 shows the graphical representations for (20) and (21), and Fig. 8 shows the corresponding plot for (23). In particular, from Fig. 7, we clearly see that the nominal Class E operation can only be maintained for a relatively low $Q$ factor, i.e., below 2 . This remains a design constraint for this symmetrically driven Class $\mathrm{E}$ amplifier, although the use of low $Q$ factors has an obvious advantage of low component stresses. Hence, in comparing the harmonic distortion performance of this amplifier with the conventional Class E amplifier, the limited choice of $Q$ factors has to be taken into consideration (see Section IV).

\section{DESIGN EXAMPLES}

Suppose $Q_{L}=1.3, Z_{o}=38.4 \Omega$, and $R=50 \Omega$. Then, from Fig. 7, $\left(\omega_{1} / \omega_{o}\right)=1.51$ and $\left(\omega / \omega_{o}\right)=1.101$. If we wish to use a switching frequency of about $1 \mathrm{MHz}$ and a convenient range of input voltage from around 15 to $32 \mathrm{~V}$, then, using Fig. 8, we 
TABLE I

DESIGN EXAMPLES WITH $Q_{L}=1.3$ AND 1.9

\begin{tabular}{lrr}
\hline Components/Parameters & \multicolumn{2}{c}{ Values } \\
\hline$Q_{L}$ & 1.3 & 1.9 \\
$R$ & $50 \Omega$ & $50 \Omega$ \\
$Z_{o}$ & $38.4 \Omega$ & $26.3 \Omega$ \\
$\omega_{1} / \omega_{o}$ & 1.51 & 1.43 \\
$\omega / \omega_{o}$ & 1.101 & 1.124 \\
$C_{1} / C$ & 0.78 & 0.96 \\
$L$ & $6 \mu \mathrm{H}$ & $4.27 \mu \mathrm{H}$ \\
$C$ & $4.06 \mathrm{nF}$ & $6.17 \mathrm{nF}$ \\
$C_{1}$ & $3.17 \mathrm{nF}$ & $5.90 \mathrm{nF}$ \\
$f_{o}$ & $1.02 \mathrm{MHz}$ & $0.98 \mathrm{MHz}$ \\
$f$ & $1.12 \mathrm{MHz}$ & $1.10 \mathrm{MHz}$ \\
$P$ & $30-150 \mathrm{~W}$ & $50-270 \mathrm{~W}$ \\
$E$ & $14.63-32.72 \mathrm{~V}$ & $13.95-32.41 \mathrm{~V}$ \\
\hline
\end{tabular}

TABLE II

ACTUAL COMPONENT AND PARAMETER VALUES USED

\begin{tabular}{lrr}
\hline Components/ \\
Parameters & \multicolumn{2}{c}{ Values } \\
\hline$Q_{L}$ & 1.3 & 1.9 \\
$R$ & $50 \Omega$ & $50 \Omega$ \\
$L$ & $6 \mu \mathrm{H}$ & $4.27 \mu \mathrm{H}$ \\
$C$ & $4.06 \mathrm{nF}$ & $6.17 \mathrm{nF}$ \\
& $(2.2\|1.0\| 1.0 \mathrm{nF})$ & $(2.2\|2.2\| 1.0 \| 1.0 \mathrm{nF})$ \\
$C_{1}$ and $C_{2}$ & $2.67 \mathrm{nF}$ & $5.40 \mathrm{nF}$ \\
& $(2.2 \| 0.470 \mathrm{nF})$ & $(2.2\|2.2\| 1.0 \mathrm{nF})$ \\
MOSFET & $\mathrm{IRF} 640$ & $\mathrm{IRF} 640$ \\
$L_{1}$ and $L_{2}$ & $400 \mu \mathrm{H}$ & $400 \mu \mathrm{H}$ \\
$f$ & $1.22 \mathrm{MHz}$ & $1.20 \mathrm{MHz}$ \\
$E$ & $15-32 \mathrm{~V}$ & $15-32 \mathrm{~V}$ \\
\hline
\end{tabular}

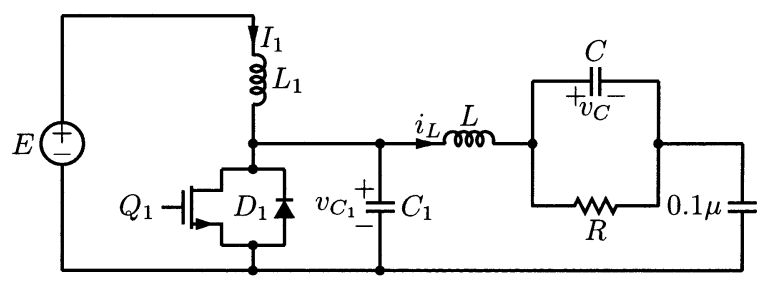

Fig. 9. Conventional single-ended Class E power amplifier (constructed for comparison).

arrive at the component and parameter values shown in Table I. The power level ranges from 30 to $150 \mathrm{~W}$, depending upon the input voltage value. Likewise, we can obtain the component and parameter values for the case $Q_{L}=1.9, Z_{o}=26.3 \Omega$ and $R=50 \Omega$, as shown in Table I. In practice, the actual component and parameter values may be chosen as shown in Table II. Also, we take the drain-to-source capacitance of MOSFETs as $500 \mathrm{pF}$.

\section{EXPERIMENTAL MEASUREMENTS}

Experiments have been carried out for the two design examples mentioned in the previous section. For the purpose of comparison, two additional conventional Class E power amplifier
TABLE III

PARAMETERS FOR CONVENTIONAL CLASS E POWER AMPLIFIER (CONSTRUCTED FOR COMPARISON) WITH $Q_{L}=1.3$ AND 1.9. OTHER VALUES SAME AS IN TABLE III

\begin{tabular}{lrr}
\hline Components/ & \multicolumn{2}{c}{ Values } \\
Parameters & \multicolumn{3}{c}{1.3} & 1.9 \\
\hline$Q_{L}$ & $1.47 \mathrm{nF}$ & $4.20 \mathrm{nF}$ \\
$C_{1}$ & $(1.0 \| 0.47 \mathrm{nF})$ & $(2.2\|1.0\| 1.0 \mathrm{nF})$ \\
& $1.36 \mathrm{MHz}$ & $1.22 \mathrm{MHz}$ \\
$f$
\end{tabular}

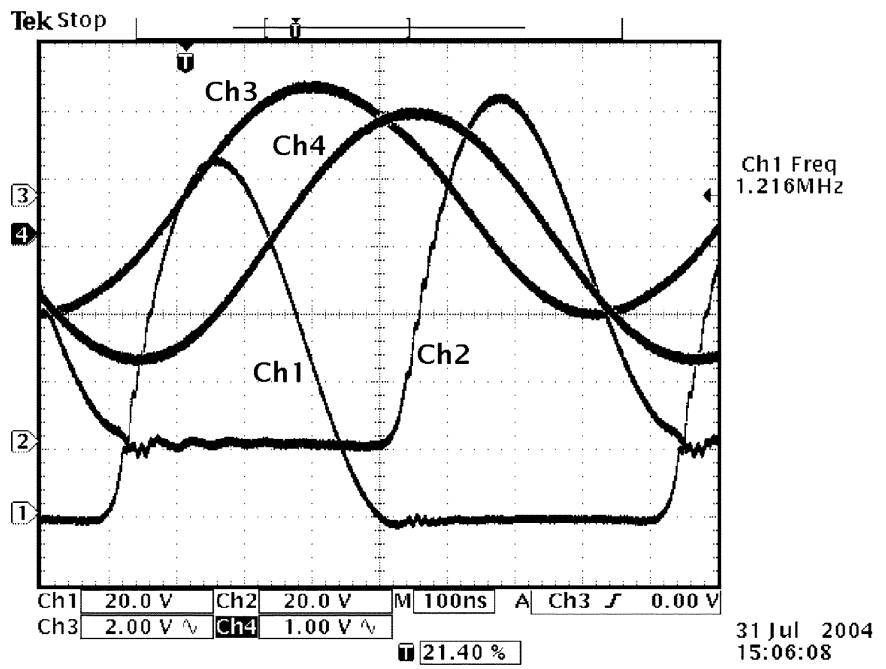

(a)

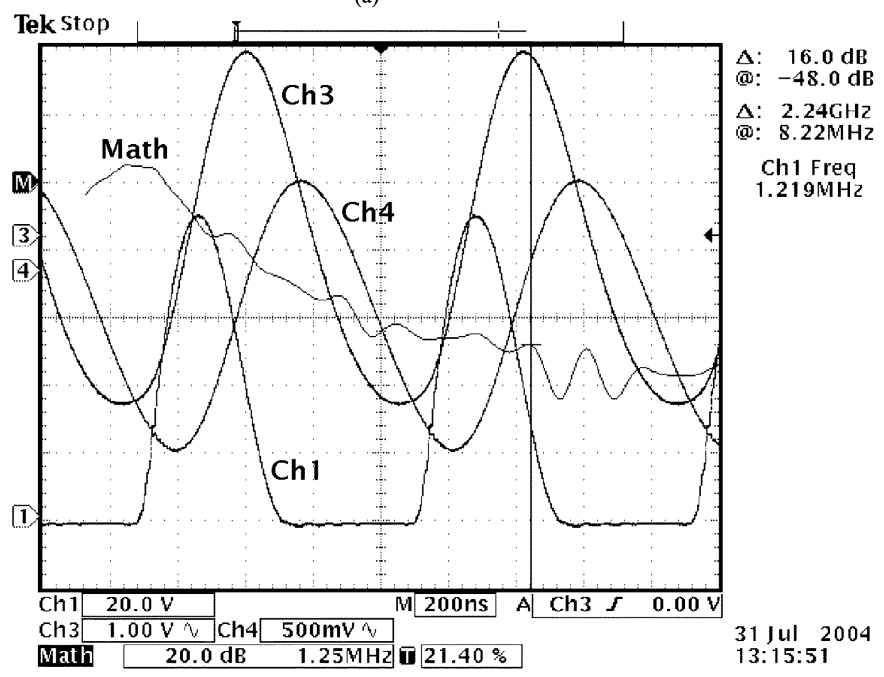

(b)

Fig. 10. (a) Waveforms from the symmetrically driven Class E power amplifier. Trace Ch1 is the voltage of $v_{C_{1}}$, trace $\mathrm{Ch} 2$ is the voltage of $v_{C_{2}}$, trace $\mathrm{Ch} 3$ is the tank inductor current and trace $\mathrm{Ch} 4$ is the load resistor current. (b) Waveforms from the original single-ended Class E power amplifier of Fig. 9 with $Q_{L}=1.9$. Trace Ch1 is the voltage of $v_{C_{1}}$, trace Ch3 is the tank inductor current and trace $\mathrm{Ch} 4$ is the load resistor current. Trace Math is the FFT of trace Ch3, and has been tabulated in Table IV. FFT for Ch4 has also been collected and tabulated in Table V.

circuits shown in Fig. 9 have also been implemented. As mentioned previously, the choice of the value of $Q_{L}$ is limited to below 2 for the symmetrically driven Class E amplifier, whereas a much larger $Q_{L}$ can be used for the conventional Class E 
TABLE IV

COMPARISON OF HARMONIC CONTENTS OF INDUCTOR CURRENT $i_{L}$

\begin{tabular}{|c|c|c|c|c|c|c|c|c|}
\hline \multirow[t]{3}{*}{ Harmonic of $i_{L}$} & \multicolumn{4}{|c|}{$Q_{L}=1.3$} & \multicolumn{4}{|c|}{$Q_{L}=1.9$} \\
\hline & \multicolumn{2}{|c|}{ Symmetrically driven } & \multicolumn{2}{|c|}{ Conventional } & \multicolumn{2}{|c|}{ Symmetrically driven } & \multicolumn{2}{|c|}{ Conventional } \\
\hline & Simulation & Measured & Simulation & Measured & Simulation & Measured & Simulation & Measured \\
\hline $2^{\text {nd }}$ & - & - & $17.07 \%$ & $16.06 \%$ & - & - & $12.53 \%$ & $12.02 \%$ \\
\hline $3^{\text {rd }}$ & $3.45 \%$ & $2.75 \%$ & $2.33 \%$ & $2.00 \%$ & $1.82 \%$ & $2.31 \%$ & $1.46 \%$ & $2.75 \%$ \\
\hline $4^{\text {th }}$ & - & - & $1.10 \%$ & $0.95 \%$ & - & - & $0.85 \%$ & $1.20 \%$ \\
\hline $5^{\text {th }}$ & $0.64 \%$ & $0.58 \%$ & $0.41 \%$ & $0.33 \%$ & $0.22 \%$ & $0.43 \%$ & $0.28 \%$ & $0.48 \%$ \\
\hline $6^{\text {th }}$ & - & - & $0.29 \%$ & $0.32 \%$ & - & - & $0.22 \%$ & $0.34 \%$ \\
\hline Total (rms) & $3.51 \%$ & $2.81 \%$ & $17.27 \%$ & $16.75 \%$ & $1.83 \%$ & $2.35 \%$ & $12.65 \%$ & $12.41 \%$ \\
\hline
\end{tabular}

TABLE $\mathrm{V}$

COMPARISON OF HARMONIC CONTENTS OF OUTPUT CURRENT $i_{o}$

\begin{tabular}{|c|c|c|c|c|c|c|c|c|}
\hline \multirow[t]{3}{*}{ Harmonic of $i_{o}$} & \multicolumn{4}{|c|}{$Q_{L}=1.3$} & \multicolumn{4}{|c|}{$Q_{L}=1.9$} \\
\hline & \multicolumn{2}{|c|}{ Symmetrically driven } & \multicolumn{2}{|c|}{ Conventional } & \multicolumn{2}{|c|}{ Symmetrically driven } & \multicolumn{2}{|c|}{ Conventional } \\
\hline & Simulation & Measured & Simulation & Measured & Simulation & Measured & Simulation & Measured \\
\hline $2^{\text {nd }}$ & - & - & $9.64 \%$ & $8.71 \%$ & - & - & $6.64 \%$ & $5.50 \%$ \\
\hline $3^{\text {rd }}$ & $1.36 \%$ & $0.91 \%$ & $0.89 \%$ & $0.91 \%$ & $0.82 \%$ & $0.76 \%$ & $0.52 \%$ & $0.78 \%$ \\
\hline $4^{\text {th }}$ & - & - & $0.32 \%$ & $0.50 \%$ & 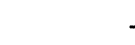 & - & $0.23 \%$ & $0.26 \%$ \\
\hline $5^{\text {th }}$ & $0.15 \%$ & $0.26 \%$ & $0.09 \%$ & $0.25 \%$ & $0.07 \%$ & $0.16 \%$ & $0.06 \%$ & $0.19 \%$ \\
\hline $6^{\text {th }}$ & - & - & $0.06 \%$ & $0.20 \%$ & 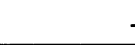 & - & $0.04 \%$ & $0.19 \%$ \\
\hline Total (rms) & $1.37 \%$ & $0.95 \%$ & $9.69 \%$ & $8.78 \%$ & $0.82 \%$ & $0.78 \%$ & $6.66 \%$ & $5.56 \%$ \\
\hline
\end{tabular}

amplifier. Obviously, a higher $Q_{L}$ gives less harmonics to the output voltage and current, and comparison of two amplifiers with different values of $Q_{L}$ must be carefully assessed (as it can be rather meaningless). In our experiments, for a fair comparison, the circuits use the same $Q$-factor and resonant tank as in the symmetrically driven Class E circuit. A large capacitor ( 0.1 $\mu \mathrm{F})$ has been added to block the dc to the output load from the asymmetrically driven resonant tank. To achieve Class E operation, the value of the parallel capacitor $C_{1}$ has been reduced and the corresponding switching frequency has been increased, as summarized in Table III.

Fig. 10(a) shows the waveforms from the symmetrically driven Class E amplifier for $Q_{L}=1.3$. The harmonic contents of the inductor current and output current are measured using the FFT function of a digital storage oscilloscope (DSO). A waveform example is shown in Fig. 10(b) for $Q_{L}=1.9$. Tables IV and $\mathrm{V}$ give comparisons of the harmonic contents of the symmetrically driven Class E amplifier and conventional single-ended Class E power amplifier. It should be noted that the even harmonics of the symmetrically driven Class E circuit are too small to be measured from the DSO and simulations. They are thus omitted in the tables. It is found that the symmetrically driven Class $\mathrm{E}$ amplifier circuit has distortion of nearly an order of magnitude lower than that of the conventional Class E circuit.

Finally, the symmetrically driven Class E amplifier, being ZVS and ZCS, is expected to demonstrate a high power efficiency. The measured efficiency from our prototypes is about $85 \%$, the loss being mainly dissipated in the heating of the inductor core and the on-resistance of the switches.

\section{CONCLUSION}

A symmetrically driven Class E power amplifier has been studied in detail. Analysis has been performed for three possible modes of operation. Nominal design equations and look-up graphs have been presented to facilitate design and implementation. Experimental circuits have been built for harmonic distortion evaluation. By virtue of the symmetrical push-pull driving voltage which cancels all even harmonics, the amplifier offers much lower harmonic distortion when compared to the conventional single-ended Class E power amplifier while maintaining similar power efficiency.

\section{ACKNOWLEDGMENT}

The authors wish to thank one of the anonymous reviewers for providing a large amount of technical information about the design of Class $\mathrm{E}$ amplifiers and the related practical problems, and to the other reviewers for their constructive comments on this work.

\section{REFERENCES}

[1] N. O. Sokal and A. D. Sokal, "Class E-A new class of high efficiency tuned singled-ended switching power amplifier," IEEE J. Solid-State Circuits, vol. 10, no. 3, pp. 168-176, Mar. 1975.

[2] N. O. Sokal et al., "Class-E high-efficiency RF/microwave power amplifiers: Principles of operation, design procedures, and experimental verification," in Analog Circuit Design: Scalable Analog Circuit Design, High-Speed D/A Converters, RF Amplifiers, J. H. Huijsing et al., Eds. Dordrecht, The Netherlands: Kluwer, 2002, pp. 269-301.

[3] J. Ebert and M. Kazimierczuk, "Class E high-efficiency tuned power oscillator," IEEE J. Solid-State Circuits, vol. SSC-16, no. 2, pp. 62-66, Feb. 1981. 
[4] M. Kazimierczuk and K. Puczko, "Exact analysis of Class E tuned power amplifier at any Q and switch duty cycle," IEEE Trans. Circuits Syst., vol. CAS-34, no. 2, pp. 149-159, Feb. 1987.

[5] H. Sekiya, I. Sasase, and S. Mori, "Computation of design values for Class E amplifiers without using waveform equations," IEEE Trans. Circuits Syst. I, Fundam. Theory Appl., vol. 49, no. 7, pp. 966-978, Jul. 2002.

[6] T. Suetsugu and M. Kazimierczuk, "Comparison of class-E amplifier with nonlinear and linear shunt capacitance," IEEE Trans. Circuits Syst. I, Fundam. Theory Appl., vol. 50, no. 8, pp. 1089-1097, Aug. 2003.

[7] B. Razavi, "Challenges in portable RF transceiver design," IEEE Circuits Dev. Mag., pp. 12-26, Sep. 1996.

[8] T. Sowlati, Y. Greshishchev, C. A. T. Salama, G. Rabjohn, and J. Sitch, "Linearized high efficiency Class E power amplifier for wireless communications," in Proc. IEEE Custom Integrated Circuits Conf., 1996, pp. 201-204.

[9] J. F. Davis and D. B. Rutledge, "A low-cost class-E power amplifier with sine-wave drive," in Dig. IEEE MTT-S Int. Microwave Symp., vol. 2, 1998, pp. 7-12.

[10] T. Kawahara and T. Suetsugu, "Class E frequency multiplier driving Class E amplifier," in Proc. Int. Telecom. Energy Conf., 1997, pp. 19-23.

[11] S. H. L. Tu and C. Toumazou, "Low-distortion CMOS complementary Class E RF tuned power amplifiers," IEEE Trans. Circuits Syst. I, Fundam. Theory Appl., vol. 47, no. 5, pp. 774-779, May 2000.

[12] M. Kazimierczuk, "Synthesis of phase-modulated dc/ac inverters and dc/dc converters," in Proc. IEE B: Elect. Power Appl., vol. 139, Jul. 1992, pp. 387-394.

[13] S. W. Ma, H. Wong, and Y. O. Yam, "Optimal design of high output power Class E amplifier," in Proc. IEEE Int. Caracas Conf. Devices, Circuits and Systems, 2002, pp. 012-1-012-5.

[14] N. O. Sokal and F. H. Raab, "Harmonic output of Class E RF amplifiers and load coupling network design," IEEE J. Solid-State Circuits, vol. SSC-12, no. 1, pp. 86-88, Feb. 1977.

[15] A. I. Zverev, Handbook of Filter Synthesis. New York: Wiley, 1967, ch. $4 \& 5$.

[16] W. K. Chen, Ed., The Circuits and Filters Handbook. New York: IEEE Press, 1995, ch. 13 \& 14.

[17] N. O. Sokal, "Unsolved theoretical problem in design of optimal lowpass filter for harmonic suppression in radio-transmitter output," IEEE Trans. Circuits Syst., vol. 27, no. 3, pp. 235-237, Mar. 1980.

[18] M. Markiewicz-Wrzeciono, "Optimal harmonic-suppression output filter for power amplifiers," in Proc. IEEE Int. Symp. Circuits and Systems, 1984, pp. 205-208.

[19] W. A. Davis and K. K. Agarwal, Radio Frequency Circuit Design. New York: Wiley, 2001, ch. 6.

[20] M. Kazimierczuk and D. Czarkowski, Resonant Power Converters. New York: Wiley Interscience, 1995, ch. 13 \& 14

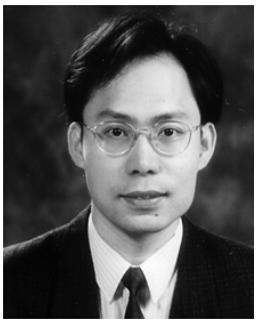

Siu-Chung Wong (M'01) received the B.Sc. degree in physics from the University of Hong Kong, Hong Kong, in 1986, the M.Phil. degree in electronics from the Chinese University of Hong Kong, in 1989, and the Ph.D. degree from the University of Southampton, U.K., in 1997.

He is currently an Assistant Professor in the Department of Electronic and Information Engineering, Hong Kong Polytechnic University, Hong Kong. His research interests include modeling of power and Internet traffic analysis.

converters, nonlinear analysis of power electronics

Dr. Wong is a member of the Electrical College, The Institution of Engineers, Australia.

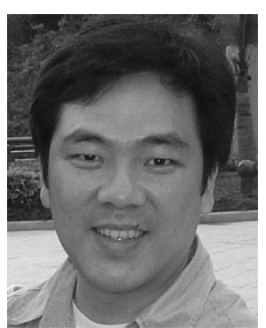

Chi K. Tse (M'90-SM'97) received the B.Eng. (Hons.) degree with first class honors in electrical engineering and the Ph.D. degree from the University of Melbourne, Australia, in 1987 and 1991, respectively.

$\mathrm{He}$ is presently a Chair Professor with Hong Kong Polytechnic University, Hong Kong, and his research interests include chaotic dynamics and power electronics. He is the author of Linear Circuit Analysis (London, U.K.: Addison-Wesley, 1998) and Complex Behavior of Switching Power Converters (Boca Raton: CRC Press, 2003), coauthor of Chaos-Based Digital Communication Systems (Heidelberg, Germany: Springer-Verlag, 2003) and Signal Reconstruction With Applications to Chaos-Based Communications (Beijing, China: Tsinghua University Press, 2005), and co-holder of a U.S. patent.

From 1999 to 2001, he served as an Associate Editor for the IEEE TRANSACTIONS ON CiRCUITS AND SYSTEMS-I: FunDAMENTAL THEORY AND APPLICATIONS, and since 1999 he has been an Associate Editor for the IEEE TRANSACTIONS ON POWER ELECTRONICS. He currently also serves as an Associate Editor of the International Journal of Systems Science, a Guest Associate Editor of the IEICE Transactions on Fundamentals of Electronics, Communications and Computers, and a Guest Editor of Circuits, Systems and Signal Processing. Since 2002, he has been appointed as Guest Professor by the Southwest China Normal University, Chongqing, China.

In 1987, he was awarded the L.R. East Prize by the Institution of Engineers, Australia. He won the IEEE Transactions on POWER Electronics Prize Paper Award for 2001 and the International Journal of Circuit Theory and Applications Best Paper Award for 2003. While with Hong Kong Polytechnic University, he received twice the President's Award for Achievement in Research, the Faculty's Best Researcher Award, the Research Grant Achievement Award and a few other teaching awards. 